\title{
Die internationale Entwicklungszusammenarbeit auf der Suche nach einem neuen Profil
}

\section{Einleitung}

Mit ihrer bald 40jährigen Geschichte hat sich die internationale Entwicklungszusammenarbeit (früher «Entwicklungshilfe») als eigenständige Branche im Gefüge der Nord-Süd-Beziehungen fest etabliert.' Ihr «Umsatz» ist stetig gestiegen und hat 1991 rund 57 Mia. Dollar erreicht. Ein beträchtlicher "Marktanteil» am «Südgeschäft", machte doch 1990 die öffentliche Entwicklungszusammenarbeit (EZA) immerhin 77 Prozent der gesamten Finanzströme aus den OECD-Mitgliedstaaten in die Entwicklungsländer aus (OECD 1992: A 87). Die Branche der EZA hat im Laufe ihrer Geschichte eigene Gesetze, Regelungsmechanismen und Organisationsstrukturen entwickelt sowie sich insbesondere eine eigene Kultur und ein entsprechendes Image aufgebaut - begleitet durch die parallel sich herausbildende eigenständige wissenschaftliche Disziplin der Entwicklungswissenschaften (vgl. MENZEL 1992 oder 1991). Nach der Aufbau-, Wachstums- und Festigungsphase ist sie nun in eine gewisse «Besinnungsphase» getreten; gerade in einer solchen Phase sind gemäß klassischen Modellen über Lebenszyklen von Branchen und Unternehmungen die Gefahren von Verkrustungen und mangelndem Veränderungs- bzw. Anpassungswillen besonders groß (z. B. aufgrund herangewachsener Altersstrukturen in Institutionen). Mit Blick auf die sich rasant verändernde weltpolitische Lage der letzten vier Jahre ist die EZA ganz besonders gefordert, ihre Ausrichtung, ihre Aufgabe, ja ihr Profil als Ganzes zu überprüfen. Nur mit genügender Weitsicht und Anpassungsfähigkeit an die großen globalen Herausforderungen hat sie eine Chance, ihre Bedeutung und Anliegen langfristig gegenüber anderen Zweigen bzw. Einflußfaktoren in den Nord-Süd-Beziehungen zu behaupten.

In diesem Artikel möchte ich aufzeigen,

- daß erstens eine Neuorientierung der internationalen EZA notwendig ist; Krisensymptome in der EZA weisen deutlich auf eine solche Notwendigkeit hin:

- daß zweitens die internationale EZA verschiedene Stärken aufweist, die oft zu wenig bekannt sind oder als solche nicht anerkannt werden;

- daß drittens die derzeitige weltpolitische Dynamik eine herausragende Chance für die internationale EZA bietet, ihre Stärken unter Beweis zu stellen und ihr Know-how sinnvoll einzubringen;

- wo viertens die Akzente einer neuorientierten EZA der Zukunft liegen könnten.

\section{Krisensymptome \\ in der internationalen Entwicklungszusammenarbeit}

\subsection{Die unermüdliche Kritik}

Seit den 80er Jahren steht die internationale EZA immer wieder im Kreuzfeuer der Kritik. An die Fundamentalkritik neoliberaler Provenienz (vgl. z. B. BAUER 1984 und 1991), wonach der Eingriff der EZA in das freie Spiel der Marktkräfte nicht nur keine, sondern sogar schädliche Wirkungen auf die Entwicklungsländer zeitige (Lähmung von Privatinitiativen usw.), hat sich die Entwicklungsbranche mittlerweile gewöhnt. Diese Kritik unterliegt - ähnlich wie bei anderen Politikbereichen mit massiven staatlichen Eingriffen zugunsten eines Wohlstandausgleichs (z. B. Regionalpolitik) - teilweise konjunkturbedingten Schwankungen, die polit-ökonomisch begründbar sind. So wird in der derzeitigen weltweiten Stagnationsphase sogar von privatwirtschaftlicher Seite die Berechtigung und Notwendigkeit staatlicher Sozialprogramme in Entwicklungsländern nicht in Frage gestellt (vgl. DE PURY 1992).

Alarmierender als die neoliberale Kritik an der EZA ist die zunehmende Resignation und Skepsis in den eigenen Reihen der EZA. Angesichts der enormen Armutsprobleme in den Entwicklungsländern macht sich bei vielen Entwicklungsexperten und -wissenschaftern Frustration und Ohnmacht breit; bei Entwicklungsagenturen schlägt sich dies in einer gewissen «Donor Fatigue» bezüglich bestimmter Regionen des Südens nieder (besonders in Afrika). Trotz jahrzehntelangen Bemühungen des «Unternehmens Entwicklungshilfe» haben Elend und Hunger in weiten Teilen des Südens zugenommen. In kritischen Beurteilungen der EZA durch Insider (ehemalige Entwicklungsexperten und -wissenschafter) ist die Rede von "Händlern der Armut» (HANCOOK 1989), "Verwaltung des Elends bzw. der Armut" (KORAMKA/KREUL 1991), «Tödlicher Hilfe» (ERLER 1985), Mittelabflußzwang (HAGEN 1988) u. a.

In jüngster Zeit wird sogar zunehmend Kritik von den Hilfeempfängern selbst laut. Die Afrikanerin A. KABOU

Christoph Graf, Dr. phil. II, Programmbeauftragter in der Direktion für Entwicklungszusammenarbeit und humanitäre Hilfe, Eidg. Dep. für auswärtige Angelegenheiten, Eigerstr. 73 . 3003 Bern 
sieht in der Drittweltbewegung des Nordens «une sorte de mouvement d'infantilisation des peuples décolonisés» (1991: 47); sie beklagt, daß die Hilfe vom Norden ohne Langfristvision, ohne Blick auf ein nachhaltiges Entwicklungsmodell und ohne genügende Rücksichtnahme auf lokale Kulturen durchgeführt werde; ihrer Meinung nach beruhen viele Entwicklungsbemühungen des Nordens auf überholten marxistischen Vorstellungen desillusionierter Entwicklungsexperten. Reichere Entwicklungsländer, die nicht mehr so stark auf ausländische Hilfsgelder angewiesen sind, kritisieren zunehmend den Stil der EZA, insbesondere die seit kurzem diskutierte politische Konditionierung von Hilfeleistungen. Zum Beispiel verzichtete die Regierung Indonesiens letztes Jahr lieber auf die EZA-Leistungen aus den Niederlanden, als sich den politischen Auflagen des Gebers im Bereich der Menschenrechte zu beugen; sie warf die holländischen Entwicklungsexperten kurzerhand aus dem Land.

\subsection{Akzeptanzprobleme in den Gebernationen}

Diese unermüdliche Kritik und die zunehmende Herkunftsvielfalt der Kritiker beeinträchtigten das Ansehen der EZA. In der Bevölkerung der Geberländer nehmen Vorbehalte gegenüber der EZA laufend zu; Effizienz und Effektivität der bisherigen EZA werden zusehends in Frage gestellt. Beispielsweise in den in Sachen EZA traditionell großzügigen skandinavischen Ländern Schweden und Norwegen oder in Kanada regt sich heute kaum Opposition gegen bereits beschlossene oder noch anstehende (teilweise massive) Budgetkürzungen bei der öffentlichen EZA; in der Schweiz beurteilten laut einer Meinungsumfrage 1991 bereits über ein Viertel aller Stimmberechtigten die Kosten der Entwicklungshilfe als untragbar, bei steigendem Trend (DEMOSCOPE 1992). Wenn auch konjunkturelle Faktoren in diesem Zusammenhang eine gewisse Rolle spielen mögen, scheint die abnehmende Akzeptanz der EZA in der Bevölkerung des Nordens doch eher struktureller Natur zu sein. Die seit längerer Zeit feststellbare Stagnation der Spendenaufkommen privater Hilfswerke weist deutlich in diese Richtung. ${ }^{2}$

Insgesamt hat die schwindende Akzeptanz der EZA bisher quantitativ noch wenig auf die Volumen der öffentlichen EZA durchgeschlagen. Die Gefahr ist jedoch groß, daß dies - mit einem gewissen Time-lag - zunehmend geschieht. Gerade in der derzeitigen Periode knapper Staatsbudgets dürfte der Posten der EZA in naher Zukunft besonders starkem Kürzungsdruck ausgesetzt sein, hat sich doch der politische Druck zur Stützung früher geopolitisch wichtiger Entwicklungsregionen im Süden nicht zuletzt aufgrund des veränderten West-Ost-Verhältnisses deutlich verringert. (Anderseits ist vor dem Hintergrund der zu bewältigenden globalen Probleme [Umweltzerstörung, Migration; vgl. Kap. 4.1] auch eine vermehrte Umorientierung der bisherigen Unterstützungsleistungen auf diese Probleme denkbar.)

\subsection{Verdrängung \\ durch andere außenpolitische Handlungsbereiche}

Obwohl die meisten staatlichen Entwicklungsagenturen der Geberländer in den Außenministerien untergebracht sind, fristeten sie bis vor kurzem ein verhältnismäßig isoliertes, von der übrigen Außenpolitik weitgehend abgekoppeltes Dasein. In vielerlei Hinsicht monopolisierten sie die Beziehungen zu den Entwicklungsländern regelrecht. Die Entwicklungsbehörden und die nichtstaatlichen Entwicklungsorganisationen hielten sich für die maßgebenden Sachfragen zuständig und kompetent und trugen damit zu einer sektoriellen Abschottung der EZA gegenüber den restlichen außenpolitischen Handlungsbereichen bei. Die Dialogbereitschaft mit Vertretern anderer Interessengruppierungen war vielfach gering (vgl. z. B. die bissige Debatte der 70er Jahre zwischen Wirtschafts- und Entwicklungskreisen über die Rolle von Multis im Entwicklungsprozeß), die Voraussetzungen für die Ausgestaltung kohärenter Außenbeziehungen waren entsprechend ungünstig.

Dies änderte sich mit der zunehmenden Globalisierung verschiedener Probleme, beispielsweise im Umwelt- und Energiebereich, in Migrations-, Flüchtlings- und Sicherheitsfragen. Andere staatliche und nichtstaatliche Stellen, die ursprünglich hauptsächlich innenpolitische Themen zu vertreten hatten (z. B. Umweltfachstellen und Naturschutzorganisationen), mischten sich zusehends in Fragen der Nord-Süd-Beziehungen ein: Die Domäne der Entwicklungsagenturen wurde von verschiedenen Fachministerien und -organisationen betreten.

Verschiedene Entwicklungsorganisationen reagierten vorerst (und reagieren teilweise heute noch) mit einer Abwehrhaltung. Erst die UNCED-Konferenz in Rio im Juni 1992 über Umwelt- und Entwicklungsfragen stellte einen gewissen Durchbruch bezüglich Kommunikation und Koordination verschiedener relevanter Instanzen dar und war für alle Beteiligten ein wichtiges Lehrstück, sich mit neuen Akteuren auf dem Gebiet der Nord-SüdBeziehungen zu verständigen, neue Dialog- und Zusammenarbeitsformen zu erproben. Die Skepsis und ein gewisses Mißrauen gegenüber neuen Akteuren auf dem Gebiet derNord-Süd-Beziehungen ist in vielen Entwicklungskreisen aber noch keineswegs ausgeräumt; viele (v. a. traditionelle) Entwicklungsorganisationen befürchten eine Schmälerung ihres Macht- und Einflußbereiches. Die mangelhafte Erfolgsbilanz der EZA dürfte sogar Ängste hervorrufen, wonach die gesamte Branche der EZA mittelfristig in Existenzgefahr schweben könnte, falls nicht rechtzeitig einschneidende Neuerungen und Anpassungen an das veränderte internationale Umfeld eingeleitet werden.

Die dargelegte Häufung von Krisenanzeichen in der EZA deutet nicht nur darauf hin, daß die Branche für ein erfolgreiches Weiterbestehen ihr Aufgabenprofil, ihr Image und ihre Kultur neu überdenken muß; die aufgeführten Krisensymptome geben auch Hinweise auf die Richtungen, in welche Änderungen gehen sollten. 


\section{3. "Vergessene» Leistungen der bisherigen Entwicklungszusammenarbeit}

\subsection{Erfolge in Entwicklungsländern}

Die dominierenden kritischen und selbstkritischen Stimmen gegenüber der EZA drängen bisweilen die positiven Leistungen der EZA in den Hintergrund. Neben Mißerfolgen sind aber in Entwicklungsländern auch beträchtliche Erfolge zu verzeichnen. So folgert CLASSEN (1990: 413) aufgrund der Auswertung von umfangreichem Material über Entwicklungsprogramme: «Der größte Teil der EZA ist hinsichtlich der gesetzten Ziele erfolgreich.» Offensichtlich hängt die Erfolgsbeurteilung in der EZA in besonders hohem Maße davon ab, wie Erfolg bzw. Mißerfolg gemessen wird. Sicherlich darf bei einer gesamthaften Erfolgsbilanzierung der EZA in Entwicklungsländern das krasse Mißverhältnis zwischen bestehendem (beinahe unendlichem) Bedarf an Entwicklungsleistungen und den zur Verfügung stehenden Mitteln der EZA nicht außer acht gelassen werden. Selbst wenn jeder EZA-Franken auf sinnvollste Weise eingesetzt würde, wäre die EZA allein nicht imstande, das Ausmaß der absoluten Armut in den armen Entwicklungsländern grundsätzlich zu verändern. (Diese Tatsache ist bei einer Neuausrichtung der EZA besonders zu beachten; siehe Kap. 5.2). Deshalb ist die Argumentation falsch, die EZA habe versagt, weil die Armut noch nicht verschwunden sei. Damit soll in keiner Weise bezweifelt werden, daß die EZA in vielen Belangen noch wesentlich verbessert werden könnte.

Oft ist es schwierig, positive Entwicklungen im Süden auf die Leistungen der EZA zurückzuführen. Es darf jedoch mit Fug und Recht angenommen werden, daß die EZA zur Verbesserung der menschlichen Lebensbedingungen in Entwicklungsländern beigetragen hat, ${ }^{3}$ fließt doch ein beträchtlicher Teil der EZA-Mittel in soziale Infrastruktur (vgl. OECD 1992: A 41 ). Einige ausgewählte - in weiten Bevölkerungs- und gewissen Entwicklungskreisen oft "vergessene» bzw. verdrängte - diesbezügliche Fakten (vgl. UNDP 1993: 12 und 1992: 24):

- Die Alphabetisierung von Erwachsenen stieg im Süden von 46 Prozent im Jahre 1960 auf 64\% im Jahre 1990; das Gefälle zum Norden wurde damit um einen Drittel reduziert.

- Die Kalorienversorgung pro Kopf nahm zwischen 1965 und 1985 um 20 Prozent zu; im gleichen Zeitraum verbesserte sich die durchschnittliche Kalorienversorgung von 90 Prozent des Gesamtbedarfs ( 1965) auf 107 Prozent (1985); zwischen 1965 und 1990 verdoppelte sich die Anzahl Entwicklungsländer mit einer täglich ausreichenden Kalorienversorgung pro Kopf.

- Die Sterblichkeit von Kindern unter 5 Jahren wurde zwischen 1960 und 1990 mehr als halbiert.

\section{2 "Nebenprodukte» der Entwicklungszusammenarbeit im Norden}

«Nebenprodukte» der EZA, welche nicht der Zielbevölkerung in Entwicklungsländern, sondern den Geberländern zugute kommen, sind in vielen Entwicklungskreisen tabu. Zu einer Leistungsbilanz der EZA gehören jedoch alle erbrachten Leistungen, auch solche, die aus entwicklungspolitischer Optik nicht unbedingt erwünscht bzw. erstrebenswert sind.

Es ist hinlänglich bekannt, daß EZA neben entwicklungspolitischen Zielsetzungen zugunsten des Südens auch durch Eigeninteressen der Geberländer geleitet wird. Die amerikanische Hilfe orientiert sich ausgesprochen stark an militärisch-strategischen Überlegungen; so wird z. B. die Militärhilfe an Israel dem amerikanischen EZABudget zugerechnet. Zudem knüpfen alle Geberländer einen Teil ihrer EZA-Mittel direkt mit Exportaufträgen zugunsten ihrer heimischen Industrie (sogenannte «gebundene Hilfe»; z. B. Mischkredite). Die Frage, inwieweit die EZA als "Marketinginstrument" für die Wirtschaft der Geberländer mißbraucht wird, bildet im Zusammenhang mit der Thematik der "gebundenen Hilfe" immer wieder Gegenstand internationaler Diskussionen (z. B. im Entwicklungsausschuß der OECD).

Auch die Schweiz profitiert vom "Geschäft» der EZA, nicht zuletzt aufgrund ihrer international insgesamt recht kompetitiven Industrie. Es wird geschätzt, daß pro Entwicklungshilfefranken des Bundes der Schweizer Wirtschaft Aufträge für rund 90 Rappen zufließen (GERSTER 1992: 18). Dies kommt einem jährlichen Auftragsvolumen für die Schweizer Wirtschaft in der Größenordnung von rund 1 Mia. Franken gleich; Aufträge und Kapitalerträge von ausländischen bilateralen und multilateralen Organisationen (Weltbank u. a.) nicht eingerechnet. Entsprechend dürften rund 100000 Arbeitsplätze direkt oder indirekt von der EZA und vom Handel mit Entwicklungsländern abhängen (CASH 1992). Die Bedeutung der "gebundenen Hilfe» ist in der Schweiz im internationalen Vergleich klein. Entsprechend dürften der direkte Nutzen und die Beschäftigungswirksamkeit des «Entwicklungsgeschäftes» in anderen Industrieländern noch bedeutsamer sein.

Damit wird deutlich, daß die Entwicklungsbranche in vielen Gebernationen einen nicht zu unterschätzenden Wirtschaftszweig darstellt. Solchen Verknüpfungen mit der einheimischen Wirtschaft ist insbesondere bei politischen Ausmarchungen über Weiterführung bzw. Ausweitung von EZA-Leistungen gebührende Beachtung zu schenken.

\subsection{Vorleistungen für die Bewältigung globaler Probleme}

Die Branche der EZA blickt auf einen reichen Erfahrungsschatz aus rund 40 Jahren Tätigkeit in und mit Entwicklungsländern zurück. Sie hat in dieser Zeit Methoden und Instrumente entwickelt und zunehmend professionalisiert, wie zusammen mit Entwicklungsländern Projekte und Programme durchgeführt werden können ${ }^{4}$ und wie 
politischer Dialog praktiziert werden kann. ${ }^{5}$ Dabei hat sich die Erkenntnis durchgesetzt, daß Aktionen nur dann dauerhafte Wirkungen erzeugen, wenn auf die lokalen Verhältnisse - in kultureller, ökologischer, sozialer und wirtschaftlicher Hinsicht - genügend Rücksicht genommen wird; eine einfache Übertragung von Wissen und Technologien vom Norden in den Süden verspricht keine nachhaltigen Resultate; es braucht vielmehr echte $\mathrm{Zu}$ sammenarbeit und Partnerschaft zwischen Nord und Süd.

Dieser Erkenntnis folgend, hat sich die EZA zunehmend lokale Kenntnisse über Entwicklungsregionen angeeignet und sich darauf spezialisiert, Probleme kontextspezifisch, partnerschaftlich (von den Bedürfnissen ausgehend) und fächerübergreifend (multidisziplinär) anzugehen. Der geringen Kaufkraft wegen waren viele Regionen des Südens, auf die sich die EZA konzentrierte, lange Zeit nur von geringem wirtschaftlichem Interesse für die Industrieländer. Erst im Zuge der Globalisierung verschiedener Problemstellungen und der Einsicht im Norden, daß das Überleben auf dem Planet Erde langfristig nur in Zusammenarbeit mit dem Süden möglich sein wird, gewinnen solche lokalen Kenntnisse über ursprünglich uninteressante Entwicklungsregionen sowie Wissen im Umgang mit Entwicklungsländern wieder an Bedeutung.

Der Erfahrungsschatz und das Know-how der EZA stellt für viele andere Branchen, die sich mit Nord-Süd-Fragen auseinandersetzen, eigentliches Pionierwissen dar, das vielfach in Verborgenheit brachliegt und in vielen Belangen bisher noch nicht optimal genutzt wird. Eine Illustration dazu: Das Postulat der Partnerschaft, wonach die Bedürfnisse der Entwicklungsländer den Interessen der Industrieländer gleichberechtigt entgegenstehen, blickt in gewissen Kreisen der EZA schon auf eine lange Tradition zurück. Die Privatwirtschaft (insbesondere multinationale Unternehmen, aber auch die Weltbank und andere große Institute der Entwicklungsfinanzierung) praktizierten demgegenüber über lange Zeit einen vom Norden bestimmten, angebotsorientierten Wissens- und Technologietransfer, dessen Konsequenzen heute entweder in unvollendeten bzw. nicht funktionierenden Infrastrukturanlagen (z. B. Kraftwerke) oder in Schuldenbergen sichtbar werden. In jüngster Zeit machen sich nun auch fortschrittliche international tätige Wirtschaftskapitäne dieses Postulat der Partnerschaft und der stärkeren Bedürfnisorientierung aus der EZA zum Vorsatz: Kooperation statt Transfer heißt jetzt die Devise, sei es im technologischen oder im Finanzbereich (vgl. SCHMIDHEINY 1992: 166 ff.).

Das vorhandene Wissen der EZA im Umgang mit Entwicklungsländern kann in gewissem Sinne als Vorleistung für die anstehende Bewältigung globaler Probleme betrachtet werden. In diesem Zusammenhang lautet die entscheidende Frage: Wie kann dieses vorhandene Wissen geeignet mobilisiert und genutzt werden?

\section{Die weltpolitische Dynamik als Chance}

\subsection{Die Bedeutung der Entwicklungsländer bei globalen Problemen}

Die seit Ende der 80er Jahre aufgekommene Dynamik bezüglich globaler Umwelt-, Energie-, Migrations-, Flüchtlings- und Sicherheitsfragen birgt für die Welt insgesamt beträchtliche Gefahren in sich. Für die EZA ergeben sich aufgrund dieser weltpolitischen Dynamik große Chancen, sich als Instrument der allgemeinen Existenzsicherung zu bewähren, indem sie nützliche Beiträge zur Beantwortung dieser globalen Fragen liefert, sind doch in ausgeprägtem Maße die Beziehungen zu den Entwicklungsländern angesprochen.

Ohne enge Zusammenarbeit zwischen Industrie- und Entwicklungsländern sind die globalen Probleme unseres Planeten nicht lösbar; Nord und Süd bilden eine untrennbare Schicksalsgemeinschaft:

- Die Lungen unserer Erde liegen in den Regenwäldern des Südens.

- Ebenso liegen die Reservoire der Artenvielfalt und damit wichtige «Rohstoffe» für die moderne Biotechnologie in den Regenwäldern des Südens.

- Der Energiebedarf wächst im Süden schneller als im Norden: In weniger als 25 Jahren dürfte der gesamte Energieverbrauch der Entwicklungsländer denjenigen der Industrieländer übertreffen.

- Ein Migrations- und Flüchtlingspotential von über 100 Mio. Personen in Entwicklungsländern drückt Richtung Norden.

- Das wachsende militärische Potential des Südens gefährdet die Sicherheitslage im Norden und für die Industrieländer überlebenswichtige natürliche Ressourcen (vgl. die Erfahrung des Golfkrieges).

Bei diesem dichten Beziehungsgeflecht zwischen Nord und Süd sind aus Sicht der EZA die Fragen interessant: Welche Rolle kommt der EZA bei der Überwindung solcher globalen Probleme zu? Wie kann die EZA relevante Erfahrung, relevantes Wissen sinnvoll als Beitrag zur Ausarbeitung von Lösungen einbringen?

\subsection{Parallelen zwischen Ost- und Südhilfe}

Nach den Umwälzungen in den Staaten Osteuropas und der ehemaligen Sowjetunion begannen die westlichen Industrieländer mit Unterstützungsprogrammen zur Erleichterung des Auf- und Umbaus dieser Staaten in Richtung Marktwirtschaft. Von der Aufgabe her sind deutliche Parallelen zwischen dieser Osthilfe und der schon rund 40 Jahre älteren Südhilfe erkennbar: Bei beiden Hilfestellungen geht es in erster Linie um den langfristigen Auf- und Umbau von Gesellschafts- und Wirtschaftsstrukturen. Wohl sind die Ausgangsbedingungen zwischen gewissen Ländern des Südens und solchen des Ostens unterschiedlich (aber wohl kaum unterschiedlicher als innerhalb der verschiedenen Regionen der sogenannten Dritten Welt). Ein Markenzeichen der EZA liegt ja genau in der Fähigkeit, sich auf sehr unterschiedliche 
Ausgangsbedingungen, eben auf lokale Gegebenheiten, einzustellen.

In der Schweiz wie auch in den meisten anderen westlichen Industrieländern ist ein Schulterschluß zwischen Süd- und Osthilfe bisher nicht geglückt, wohl erstens aus Befürchtungen seitens der traditionellen Agenturen der Südhilfe heraus, daß eine markante Umleitung von Entwicklungshilfegeldern Richtung Osten stattfinden könnte, zweitens wohl aufgrund der Zweifel aus Politik- und Wirtschaftskreisen an den bisherigen Erfolgen der traditionellen Südhilfe und drittens wohl aus der Überzeugung heraus, daß Unterstützungsmaßnahmen zugunsten des Ostens etwas grundlegend anderes sind als Südhilfe (Stichwort: Transitionsländer). So werden viele alte Fehler aus den Ursprüngen der EZA heute neu wieder im Rahmen der Osthilfe begangen (z. B. Gießkannen- statt Konzentrationsprinzip): Ein Erfahrungstransfer oder gar ein kooperativer Dialog zwischen Ost- und Südhilfe findet bis heute nur sehr spärlich statt (vgl. NZZ 1992). Das Beispiel der Osthilfe zeigt auf, daß es der EZA noch kaum gelungen ist, ihre Fähigkeiten und ihr Know-how einem breiteren Nutznießerkreis zugänglich zu machen. Die diesbezügliche Bewährungschance wurde leider bisher zu wenig wahrgenommen.

Im Zusammenhang mit der Dynamik globaler Probleme (vgl. Kap. 4.1) kann heute noch kaum abgeschätzt werden, inwieweit die EZA ihre diesbezüglichen Chancen wahrgenommen hat, sich mit relevantem Wissen zu bewähren. Hingegen bleibt hinsichtlich des künftigen Aufgabenprofils der EZA wichtig, daß der Nutzung solcher Chancen mehr Beachtung geschenkt werden sollte.

\section{Neue Akzente für die künftige Entwicklungszusammenarbeit}

\subsection{Klares Zielverständnis als Voraussetzung}

Obige Ausführungen verdeutlichen gewisse Orientierungsschwierigkeiten und eine gewisse Trägheit der internationalen EZA: Auf die vielschichtigen Krisensymptome (vgl. Kap. 2) reagiert die EZA-Branche bisher vorwiegend mit einer Defensivhaltung, aber kaum mit neuen Impulsen und Innovationen; es gibt bis anhin kaum erkennbare Kräfte innerhalb der EZA, welche das eher negativ verzerrte Bild über die bisherigen Leistungen der EZA zu korrigieren versuchten (vgl. Kap. 3); die sich für die EZA bietenden Profilierungschancen aufgrund weltpolitischer Veränderungen werden kaum als solche wahrgenommen, geschweige denn in genügendem Maße als solche genutzt (vgl. Kap. 4). Diese Schwächen hängen mit der mangelnden Klarheit über die strategische Zielausrichtung der EZA zusammen.

Die EZA ist eines unter mehreren außenpolitischen Instrumenten der Geberländer. Jedes dieser außenpolitischen Instrumente verfolgt eine primäre Zielsetzung (z. B. zielen außenwirtschaftspolitische Instrumente wie Exportrisikogarantien in erster Linie auf die Förderung nationaler Wohlfahrt ab, während z. B. Instrumente der Umweltaußenpolitik wie Emissionsabgaben hauptsächlich auf die Erhaltung natürlicher Ressourcen ausgerichtet sind). Die oberste Zielsetzung der EZA liegt in der konsequenten Förderung der internationalen sozialen Gerechtigkeit. "Obwohl diese Zielsetzung oft recht klar in Gesetzen verankert ist (so auch in der schweizerischen Gesetzgebung ${ }^{9}$ ), wird sie in der Realität der EZA häufig mit anderen Zielen vermischt, je nach den dahinter stehenden Interessen. Beispielsweise sollte die EZA in den Augen der Industrie auch Exportinteressen, in den Augen von Nationalisten auch der Fernhaltung potentieller Immigranten usw. dienen.

Aus dieser obersten, strategischen Zielsetzung leitet sich die Hauptaufgabe der EZA ab: die Verbreitung und Förderung "globaler Sozialpolitik». In der Vergangenheit kam dieser Auftrag der EZA in vielen Entwicklungskreisen als falsch verstandene Helfermentalität zum Ausdruck. Die Förderung "globaler Sozialpolitik» heißt aber nicht in erster Linie, Almosen verteilen, sondern initiative und produktive Kräfte unterstützen (die Zusammenarbeit mit dem privaten Sektor ist dabei eingeschlossen) sowie Voraussetzungen schaffen, die benachteiligte Bevölkerungsgruppen dazu befähigen, ihre Lage eigenständig zu verbessern («empowerment»). Die Förderung "globaler Sozialpolitik» heißt auch, jede Art von Aktivität in Nord und Süd möglichst in eine sozialverträgliche Richtung lenken. Ein so verstandener Auftrag der EZA erfordert nicht nur das Ernstnehmen der Kohärenzaufgabe (gegenseitige Abstimmung verschiedener Politikbereiche), sondern auch die klare Erkenntnis, daß die Aufgaben der EZA aus zwei ebenbürtigen Komponenten bestehen: einer nach außen orientierten (außenpolitischen) Komponente mit konkreten Aktionen in und mit Entwicklungsländern sowie einer nach innen orientierten (innenpolitischen) Komponente mit Aktionen in und mit Geberländern. Natürlich sind beide Komponenten eng miteinander verknüpft.

\subsection{Politikdialog mit dem Süden}

Die bisherige EZA sah ihre Aufgabe bis anhin vor allem in der Durchführung von Aktionen in und mit Entwicklungsländern und konzentrierte ihre Kräfte demzufolge hauptsächlich auf die nach außen orientierte Komponente. Dabei dominierte lange Zeit punktuelle Projekthilfe unter weitgehender Vernachlässigung von Makround Mesoebene. Erst mit der wachsenden Einsicht, daß einzelne Projektaktivitäten unter schlechten Rahmenbedingungen keine eigenständigen Multiplikator- und damit Breiteneffekte hervorrufen können und daß «Entwicklung» auf breiter Basis kaum mit den beschränkten Mitteln des Nordens für einzelne, unkoordinierte Projekte erzeugt werden kann, entstanden Ansätze der Programmhilfe ${ }^{10}$ und des Politikdialoges."

Während Programmhilfe bereits einen wesentlichen Schritt in die richtige Richtung darstellt, dürfte auf lange Sicht Politikdialog die nachhaltigste Form der Zusammen- 
arbeit mit Entwicklungsländern sein. Denn in einem solchen Gedankenaustausch zwischen Geber- und Empfängerländern

- wird das gegenseitige Verständnis für die vorgebrachten Probleme erhöht;

- wird die gegenseitige Sicht über realistische Möglichkeiten und Grenzen der verschiedenen Formen von EZA und anderen Austauschbeziehungen geschärft;

- tritt die Verantwortung des Staates und die Bedeutung politischen Handelns klar hervor.

Im derzeitigen Politikdialog steht hauptsächlich das Verhalten der Empfängerländer zur Diskussion. Mit Blick auf die dichte Vernetzung vieler Probleme zwischen Nord und Süd ist künftig anzustreben, daß auch Politiken und Verhaltensweisen der Geberländer in bezug auf entwicklungsländerrelevante Themen Gegenstand eines solchen Dialogs werden.

Die Qualität des Politikdialogs hängt wesentlich vom einbringbaren Erfahrungspotential der Gesprächspartner ab. Geber sind deshalb auf eigene Projekterfahrungen aus dem Bereich der technischen Zusammenarbeit angewiesen; ansonsten verlieren sie an Glaubwürdigkeit und Kompetenz. In diesem Sinne hat die bilaterale technische Zusammenarbeit eine unverzichtbare Zuträgerrolle für den Politikdialog. Kurz gesagt ist technische Zusammenarbeit im Dienste des Politikdialogs notwendig, per se aber kaum mehr gerechtfertigt.

Eine solche Umorientierung der EZA in Richtung Politikdialog stellt eine wichtige künftige Aufgabe dar. Auch wenn ein solcher Umorientierungsprozeß in Ansätzen schon begonnen hat, so ist das Image und auch die interne Kultur der gegenwärtigen EZA in weiten Teilen noch stark von einem punktuellen Projektdenken geprägt.

\subsection{Förderung der Weltverträglichkeit des Nordens}

Eine nach innen orientierte, innenpolitische Komponente der EZA ist - entsprechend der oben erörterten Dominanz von entwicklungsländerbezogenem Projektdenken - bisher in Entwicklungskreisen nur ungenügend entwikkelt. Für Informations- und Sensibilisierungszwecke im Inland setzt beispielsweise die öffentliche EZA der Schweiz weniger als ein halbes Prozent ihres Budgets ein. Eine solche krasse Vernachlässigung der Innenpolitik ergab sich trotz des Wissens, daß das Verhalten des Nordens für den Süden beträchtliche Kosten verursacht: So beziffert der Human Development Report 1992 den Schaden für den Süden aufgrund der Abschottung des Nordens bzw. aufgrund "unvollkommener Weltmärkte» mit rund $500 \mathrm{Mia}$. Dollar, dem rund 10 fachen der weltweiten öffentlichen EZA-Leistungen. ${ }^{12}$ Die Schweiz gibt allein für ihren Agrarprotektionismus (u. a. für die Verhinderung von Agrarimporten aus Entwicklungsländern) rund $7 \mathrm{mal}$ mehr aus als für die staatliche EZA. Angesichts dieses gewichtigen Anpassungsbedarfs im Norden in Richtung welt- und insbesondere südverträgli- che Strukturen ist wenig verständlich, daß sich die EZA nicht stärker nationale Innenpolitik in den Geberländern zur Aufgabe macht und entsprechend auch Mittel dafür einsetzt. Die lange praktizierte Abschottung der EZA gegenüber anderen Politikbereichen ist heute überholt; eine Öffnung tut not. Die Verantwortlichen der EZA sollten sich vermehrt dafür engagieren, daß entwicklungspolitische Anliegen und damit eine gewisse "Südsicht» in andere Politikbereiche aufgenommen werden, z. B. in die Umwelt-, Energie-, Außenwirtschaft, Wissenschaftspolitik usw. Die Schaffung südverträglicher Strukturen im Norden dürfte in der langen Frist wirksamer und erst noch deutlich kostengünstiger für alle Beteiligten sein als eine Weiterführung bzw. Erweiterung der traditionellen, nach außen orientierten EZA in Entwicklungsländern. In der Schweiz haben Entwicklungskreise verschiedene, bisher allerdings noch zaghafte Versuche in Richtung einer solchen nach innen orientierten EZA unternommen (vgl. z. B. die Arbeit der Erklärung von Bern oder die kürzliche Ausarbeitung eines Strukturanpassungsprogramms für die Schweiz aus Sicht eines Afrikaners ${ }^{13}$ u. a.). Die innenpolitische Komponente der EZA muß in Zukunft in Form von Sensibilisierungsarbeit in den Geberländern - angefangen in den Schulen bis zur Durchführung nationaler Konferenzen über eine kohärente Südpolitik - deutlich verstärkt werden. Nur dann wirkt die EZA in ihren Bemühungen glaubhaft, wirklich einen nachhaltigen und breitenwirksamen Lösungsbeitrag zugunsten der Entwicklungsländer an die Bewältigung der anstehenden Probleme im Nord-Süd-Kontext leisten zu wollen.

\subsection{Weitergeben von Erfahrungen}

Die bessere Nutzbarmachung ihres reichen Erfahrungsschatzes und des Know-hows für andere entwicklungsländerrelevante Branchen stellt eine weitere Herausforderung für die EZA der Zukunft dar. Sie ist eng verknüpft mit dem bereits erwähnten Postulat einer verstärkten Öffnung der EZA zu anderen Akteuren auf dem Gebiet der Nord-Süd-Beziehungen (vgl. Kap. 3.3 und 5.3).

Verstärkte Bemühungen zur Verbreitung und Aufbereitung von Wissen der EZA zugunsten von Themen mit globaler Bedeutung dürften mittelfristig auch das Image und die Ausstrahlungswirkungen der Entwicklungsbranche positiv beeinflussen: Der durch die anhaltende Kritik heraufbeschworene Skeptizismus gegenüber und innerhalb der EZA könnte mit Hilfe solcher Bemühungen in Optimismus und verstärktes Selbstvertrauen auf die eigenen Stärken umschlagen.

Der Inhalt des Artikels liegt in der Verantwortung des Autors und deckt sich nicht zwangsläufig mit der offiziell vertretenen Haltung der Direktion für Entwicklungszusammenarbeit und humanitäre Hilfe. 


\section{Anmerkungen}

Zu internationaler Entwicklungszusammenarbeit werden hier sowohl langfristige Aufbauhilfe als auch kurzfristige humanitäre Einsätze (technische und finanzielle Unterstützung) gezählt.

Die Jahresversammlung europäischer Hilfswerke in Brüssel am 1./2. Dezember 1992 bestätigte solche Erosionstendenzen in der internationalen EZA: Von drohender Stagnation, einem Abbau der Entwicklungshilfe, Zunahme der Fremdenskepsis und -ablehnung in fast allen europäischen Ländern war die Rede (HEINIGER 1992)

${ }^{3}$ In einigen Sektoren ist ein solcher Zusammenhang einfacher darzulegen als in anderen: Zum Beispiel trug die von Entwicklungsgeldern finanzierte internationale Agrarforschung wesentlich zur markanten Steigerung der durchschnittlichen Weltnahrungsmittelproduktion bei.

${ }^{4}$ Zum Beispiel weisen die Planungsmethoden in Projekten derEZA in vielen Belangen einen höheren Standard auf als in der heimischen Orts- und Regionalplanung: Während die Evaluation von Planungsprozessen und -resultaten in der Regionalplanung eine vergleichsweise junge Erscheinung darstellt, ist diese in der EZA seit langem institutionalisiert.

${ }^{5}$ Vgl. koordinierte Geberkonferenzen, z. B. die Konsultativgruppen der Weltbank zwischen Geber- und Empfängerländern.

${ }^{6}$ Eine eingehendere Behandlung ökologischer Themen im Nord-Süd-Kontext befindet sich in MEADOWS 1992 FRITSCH 1990, SCHMIDHEINY 1992, HAUSER 1990/91, HEIN 1991 und VON WEIZSÄCKER 1990; zu Migrations-. Flüchtlings- und sicherheitspolitischen Themen siehe BÄCHLER 1992, MATTHIES 1992, STRAUBHAAR 1992

In den Ländern Osteuropas dürften aufgrund kultureller Ähnlichkeiten zu Westeuropa ohnehin einfachere Ausgangsbedingungen für die westliche Hilfe vorliegen als in manchen Staaten des Südens.

${ }^{8}$ Diese oberste Zielsetzung weist einerseits große Komplementaritäten zu solchen anderer außenpolitischer Bereiche anderseits aber auch deutliche Zielkonflikte auf. Mit Blick auf eine effiziente und wirksame Politikausgestaltung sind bei Komplementaritäten die sich ergebenden Synergien optimal zu nutzen, Zielkonflikte hingegen offen darzulegen.

${ }^{9}$ Vgl. dazu das Bundesgesetz über die internationale Entwicklungszusammenarbeit und humanitäre Hilfe vom 19. März 1976, Art. 2 und 5.

${ }^{10}$ Programmhilfe beinhaltet die Förderung sektoral und/ oder regional abgrenzbarer Entwicklungsvorhaben; dabei sind die Entwicklungsgelder i. d. R. nicht an spezifische Projekte gebunden.

"Der Ausdruck "Politikdialog" bezeichnet den Gedankenaustausch zwischen Geber- und Empfängerländern über die politischen Rahmenbedingungen von Entwicklungsleistungen, über die Einflußnahme der Beteiligten auf die Resultate von Entwicklungshilfeleistungen sowie über deren volkswirtschaftliche Auswirkungen.

${ }^{12}$ Eine zusammenfassende und übersichtliche Darstellung dieser Kosten ist in epd-Entwicklungspolitik 8/92 (April), Seite $20 \mathrm{ff}$., zu finden.

${ }^{13}$ Dies war ein Auftrag der Arbeitsgemeinschaft der Hilfswerke Swissaid/Fastenopfer/Brot für alle/Helvetas/Caritas an den Ökonomen E. Mireku aus Ghana. Das verfaßte PolicyPaper "Strukturreformen für die Schweiz" ist in i3W Nr. 9/92 abgedruckt.

\section{Literatur}

BÄCHLER, G. (Hrsg.; 1992): Friedens- und Konfliktforschung in Zeiten des Umbruchs, Zürich/Chur.

BAUER, P. T. (1991): The Development Frontier, London.

BAUER, P. T. (1984): Reality and Rhetoric, London.

CASH (1992): Nehmen ist seliger denn geben, Nr.30, 24. Juli, 3

CASSEN. R. (1990): Entwicklungszusammenarbeit. Fakten Erfahrungen - Lehren, dt. Ausgabe, Bern und Stuttgart.

DE PURY,D. (1992): Wandel zu Demokratie und Marktwirtschaft in Entwicklungsländern, Vortragsmanuskript, 1. Dezember, Zürich.

DEMOSCOPE (1992): Umfrage über politisches Verhalten in der Schweiz (mimeo).

ERLER, B. (1985): Tödliche Hilfe, Freiburg i. Br.

FRITSCH, B. (1990): Mensch - Umwelt - Wissen, Stuttgart.

GERSTER, R. (1992): Erhöhung der Entwicklungshilfe: Mehr als Lippenbekenntnisse? In: Informationsdienst 3. Welt, Dokument Nr. 2, Juni, Bern.

HAGEN, T. (1988): Wege und Irrwege der Entwicklungshilfe, Zürich.

HANCOOK, G. (1989): Händler der Armut. Wohin verschwinden unsere Entwicklungsmilliarden? dt. Ausgabe, München. HAUSER, J. A. (1990/91): Bevölkerungs- und Umweltprobleme der Dritten Welt, Bd.1 u. 2, Bern und Stuttgart.

HEIN, W. (Hrsg.; 1991): Umweltorientierte Entwicklungspolitik, Hamburg.

HEINIGER, M. (1993): Spart Westeuropa auf Kosten der Dritten Welt? In: Partnerschaft, Helvetas, Nr.131, 18-19.

KABOU, A. (1991): Et si l'Afrique refusait le développement? Paris.

KROMKA, F./KREUL,W. (1991): Unternehmen Entwicklungshilfe. Samariterdienst oder die Verwaltung des Elends? Zürich.

MATTHIES, V. (Hrsg.; 1992): Kreuzzug oder Dialog. Die Zukunft der Nord-Süd-Beziehungen, Bonn.

MEADOWS, D. u. D./RANDERS, J. (1992): Die neuen Grenzen des Wachstums, dt. Ausgabe, Stuttgart.

MENZEL, U. (1992): Das Ende der Dritten Welt und das Scheitern der großen Theorien, Frankfurt.

MENZEL, U. (1991): Geschichte der Entwicklungstheorie. Einführung und systematische Bibliographie, Hamburg.

MIREKU, E. (1992): Strukturreformen für die Schweiz. In: Informationsdienst 3. Welt, Dokument Nr. 9, September, Bern.

NZZ (1992): Mit der Gießkanne durch Osteuropa? 19. Mai, Nr.115, 41

OECD (1992): Coopération pour le développement. Rapport 1992, Paris

SCHMIDHEINY, S. (1992): Kurswechsel. Globale unternehmerische Perspektiven für Entwicklung und Umwelt, München.

STRAUBHAAR, T. (1992): Die neue Völkerwanderung. Treck nach Westen und Norden. In: Der Monat 4

UNDP (1993): Human Development Report, New York. UNDP (1992): Human Development Report, New York

VON WEIZSÄCKER,E.U. (1990): Erdpolitik. Ökologische Realpolitik an der Schwelle zum Jahrhundert der Umwelt, Darmstadt. 